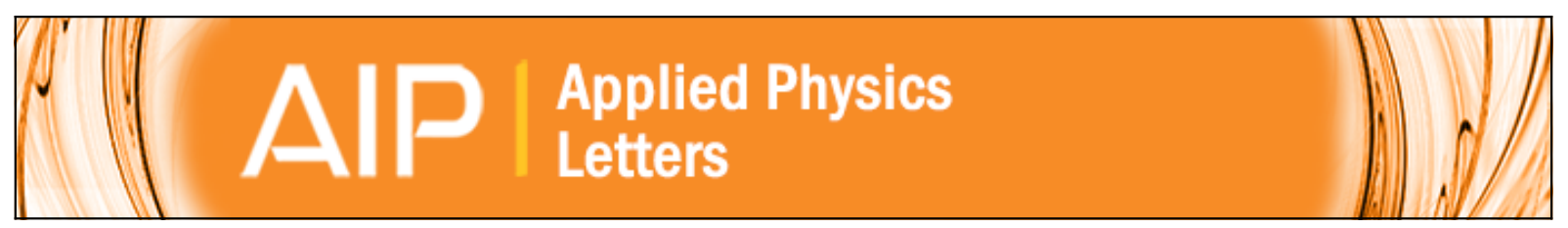

\title{
Contact resistivity and suppression of Fermi level pinning in side-contacted germanium nanowires
}

Maria M. Kolenik-Gray, Tarek Lutz, Gillian Collins, Subhajit Biswas, Justin D. Holmes, and Vojislav Krsti

Citation: Applied Physics Letters 103, 153101 (2013); doi: 10.1063/1.4821996

View online: http://dx.doi.org/10.1063/1.4821996

View Table of Contents: http://scitation.aip.org/content/aip/journal/apl/103/15?ver=pdfcov

Published by the AIP Publishing

\section{Articles you may be interested in}

Fermi-level depinning and contact resistance reduction in metal/n-Ge junctions by insertion of W-encapsulating Si cluster films

Appl. Phys. Lett. 104, 062105 (2014); 10.1063/1.4864321

Impact of fluorine treatment on Fermi level depinning for metal/germanium Schottky junctions Appl. Phys. Lett. 99, 253504 (2011); 10.1063/1.3666779

Contact resistivity and Fermi-level pinning in n-type Ge contacts with epitaxial Si-passivation Appl. Phys. Lett. 98, 013504 (2011); 10.1063/1.3530437

The influence of Fermi level pinning/depinning on the Schottky barrier height and contact resistance in $\mathrm{Ge} / \mathrm{CoFeB}$ and $\mathrm{Ge} / \mathrm{MgO} / \mathrm{CoFeB}$ structures

Appl. Phys. Lett. 96, 052514 (2010); 10.1063/1.3285163

Fermi-level pinning and charge neutrality level in germanium

Appl. Phys. Lett. 89, 252110 (2006); 10.1063/1.2410241

\section{A.P $\left.\right|_{\text {Applied Physics }} ^{\text {Journal of }}$}

Journal of Applied Physics is pleased to announce André Anders as its new Editor-in-Chief 


\title{
Contact resistivity and suppression of Fermi level pinning in side-contacted germanium nanowires
}

\author{
Maria M. Koleśnik-Gray, ${ }^{1,2}$ Tarek Lutz, ${ }^{1,2}$ Gillian Collins, ${ }^{2,3}$ Subhajit Biswas, ${ }^{2,3}$ \\ Justin D. Holmes, ${ }^{2,3}$ and Vojislav Krstić ${ }^{1,2,4, a)}$ \\ ${ }^{1}$ School of Physics, Trinity College Dublin, College Green, Dublin 2, Ireland \\ ${ }^{2}$ Centre for Research on Adaptive Nanostructures and Nanodevices (CRANN), and AMBER at CRANN, Trinity \\ College Dublin, College Green, Dublin 2, Ireland \\ ${ }^{3}$ Department of Chemistry, University College Cork, Cork, Ireland \\ ${ }^{4}$ Chair for Applied Physics, FAU Erlangen-Nuremberg, 91058 Erlangen, Germany
}

(Received 20 May 2013; accepted 5 September 2013; published online 8 October 2013)

\begin{abstract}
Electrical properties of contact-interfaces in germanium nanowire field effect transistor devices are studied. In contrast to planar bulk devices, it is shown that the active conduction channel and gate length extend between and underneath the contact electrodes. Furthermore, direct scaling of contact resistivity and Schottky barrier height with electrode metal function is observed. The associated pinning parameter was found to be $\gamma=0.65 \pm 0.03$, which demonstrates a significant suppression of Fermi level pinning in quasi-one-dimensional structures.
\end{abstract}

(C) 2013 AIP Publishing LLC. [http://dx.doi.org/10.1063/1.4821996]

The electrical performance of semiconductor nanowire (NW) devices has been put under scrutiny for over a decade. ${ }^{1-5}$ Properties of electrode/NW contact-interfaces are of particular interest ${ }^{6-10}$ due to the potential application of quasi-one-dimensional materials into future nanosensing and nanoelectronic devices. ${ }^{11}$ It has been proposed by theory that Fermi level pinning effects, which frequently hinder the performance of planar devices, ${ }^{12}$ are expected to be strongly reduced in side contacts to NW channels due to their confined geometry. ${ }^{13,14}$ Although several studies regarding the contact-resistivity in semiconducting NW devices can be found, ${ }^{6-10}$ an analysis addressing both the contact-interface properties and also verifying the plausibility of common bulk-based extrapolation methods is to-date not presented.

Germanium NWs provide an excellent basis for such a study. Since contacts to planar Ge have been demonstrated to exhibit strong Fermi level pinning, ${ }^{15,16}$ the effects of confined geometry on band realignment are expected to be most prominent in this semiconductor material.

Here, we show a comprehensive study on side contacted Ge NW devices. First, the electrically active conduction channel and gate length are studied in order to provide accurate evaluation of intrinsic transport parameters. On that basis, the device characteristics are analyzed, and scaling of specific contact resistivity and Schottky barrier height with electrode work function is shown.

Ge NWs were synthesized from Au nanoparticles catalysts via supercritical fluid-liquid-solid (SFLS) method. Details of SFLS technique can be found in earlier works. ${ }^{17,18}$ Two types of Ge NWs grown at different conditions ${ }^{19}$ were used (referred to as "type A"17 and "type B" (Ref. 18)) to demonstrate independence from the Ge NW synthesis conditions. High resolution transmission-electron-microscopy (TEM) (Fig. 1(a)) confirmed that both NW types were

\footnotetext{
a) Author to whom correspondence should be addressed. Electronic mail: krsticv@tcd.ie and vojislav.krstic@fau.de
}

monocrystalline, with few structural defects and covered by a 2-4 nm thin low-density amorphous oxide layer.

For electrical characterization, the NWs were deposited onto $\mathrm{SiO}_{2} / \mathrm{Si}$ substrates and contacted via electron beam lithography in multiterminal configuration (Fig. 1(b)). The separation between neighbouring electrodes $s$ varied from 700 to $3000 \mathrm{~nm}$ and the contact width $\Delta$ was between 350 and $600 \mathrm{~nm}$ for individual devices. Electrode height was set at $80 \mathrm{~nm}$ to ensure continuous NW coverage and to avoid surface scattering effects in the electrodes. ${ }^{20}$ Different metals
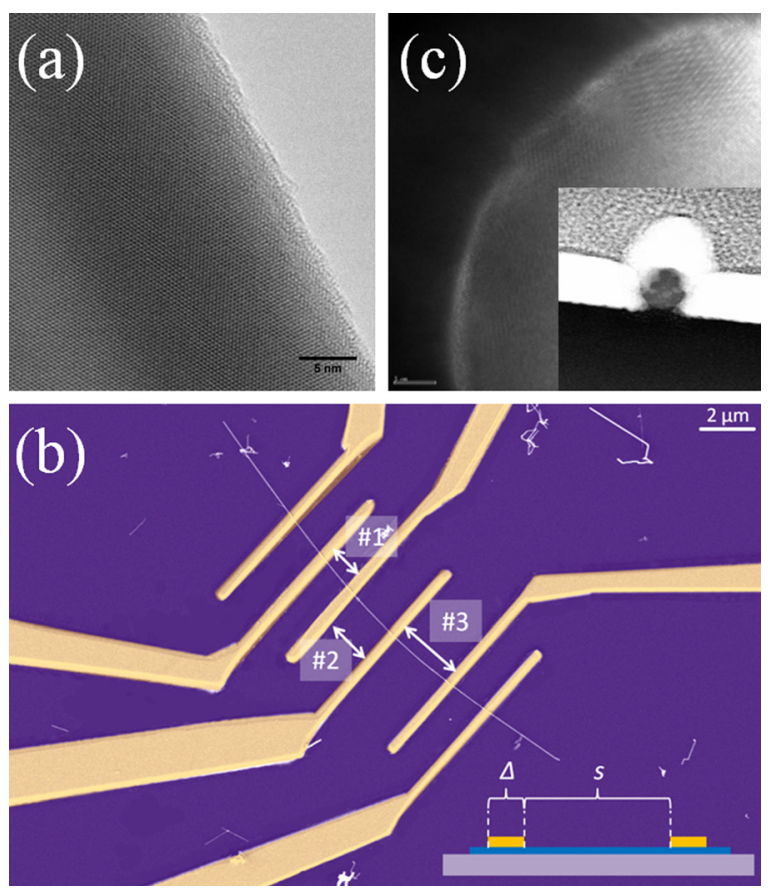

FIG. 1. (a) TEM image of a Ge NW covered by $3 \mathrm{~nm}$ amorphous oxide layer. (b) SEM image of a six-terminal device layout with varied electrode separation $s(\# 1, \# 2$, \#3, shown for a $41 \mathrm{~nm}$ Pt-contacted NW). Inset (b) NW device diagram (side-view) with electrodes of width $\Delta$ separated by distance s. (c) TEM image of a cross-section of an Au-contacted $65 \mathrm{~nm}$ Ge NW. Inset (c) Zoom-out of main figure. 
$(\mathrm{Pd}, \mathrm{Pt}, \mathrm{Au}$, and $\mathrm{Ag}$ ) were selected to account for work function differences. ${ }^{21}$

Cross-sectional lamellas of the electrode/NW regions were prepared using focused ion beam to study the quality of the contact-interfaces via TEM (Fig. 1(c)). Energydispersive X-ray spectroscopy of the contact-interface region showed the absence of oxide or contaminants for all electrode materials. ${ }^{19}$

Two- and four-terminal electrical measurements of the Ge NW devices were taken between electrodes with varied electrode-separations. The transfer characteristics (backgated) of all wires tested showed a $p$-type field effect behaviour. Based on four-point and transfer measurements, ${ }^{19}$ the NW resistivity, $\rho_{N W} \propto L_{C}^{-1}$, and mobility, $\mu_{N W} \propto L_{G}^{2} / L_{C}$, are obtained. The extracted values depend therefore on the electrically active conduction-channel, $L_{C}$, and the gate-length, $L_{G}$. To determine the correct $L_{C}$, the material-intrinsic $\rho_{N W}$ is used as it must be independent of the sample geometrythat is, independent of the separation of contacting electrodes. In Fig. 2(a), $\rho_{N W}$ is calculated for three assumed $L_{C}$ values. The analysis shows unambiguously that $\rho_{N W}$ is the same independent of the electrode separation along a single NW only if $L_{C}=s+2 \Delta$ is considered. Similarly, the active gate length $L_{G}$ can be found when addressing the mobility, and was found to be commensurate with $L_{C}$ (Fig. 2(b)). This is a counterintuitive and highly non-trivial result, as the assumption $L_{C}=s=L_{G}$ leads to resistivity overestimate and mobility underestimate, becoming more dramatic the higher the $\Delta / s$ ratio.

In our devices, values for $\rho_{N W}$ were found to range from 0.03 to $4.1 \Omega \mathrm{m}$, whereas $\mu_{N W}$ varied between 0.08 to 390 $\mathrm{cm}^{2} \mathrm{~V}^{-1} \mathrm{~s}^{-1}$. The carrier concentration, $N_{d}$, was found to vary from $2 \times 10^{14}$ to $3.2 \times 10^{18} \mathrm{~cm}^{-3}$ and was interpreted as the majority carrier (dopant) density since the measured values are well above the intrinsic concentration in $\mathrm{Ge}$ $\left(2.4 \times 10^{13} \mathrm{~cm}^{-3}\right) .{ }^{21} \rho_{N W}$ was significantly higher, while $\mu_{N W}$ lower than previously reported for bulk Ge at the same doping levels. ${ }^{22}$
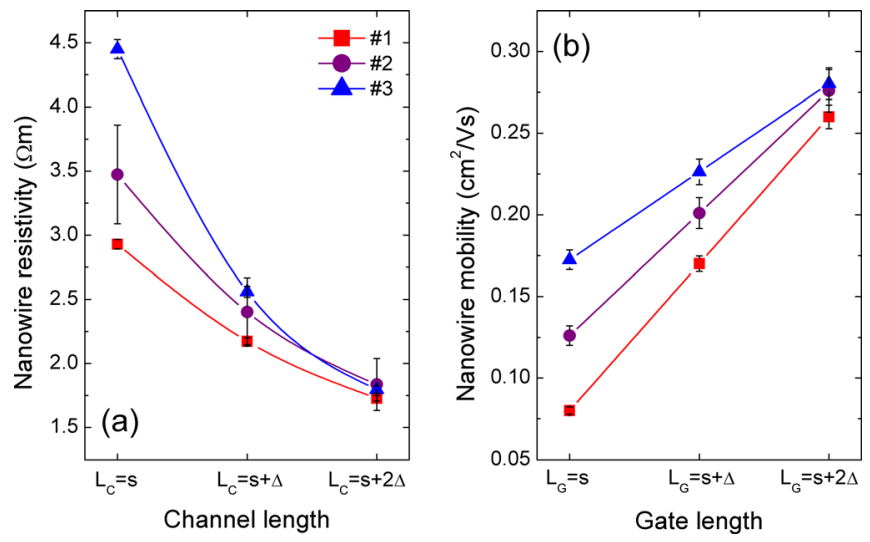

FIG. 2. (a) NW resistivity values extracted from measurement across electrode pairs with different separation (\#1-3, see Fig. 1(c)) for three different channel lengths. The values coincide for all probing positions when $L_{C}=s+2 \Delta$. (b) Field-effect mobility estimates extracted from transfer measurement across electrode pairs with different separations (analogously to (a)). Values coincide for the case of $L_{G}=s+2 \Delta=L_{C}$. Data are shown for a $60 \mathrm{~nm}$ Pd-contacted Ge NW.
To address the work function dependence of the specific contact resistivity, $\rho_{C}$, the total nanodevice resistance, $R_{t o t}$, needs to be considered first. $R_{t o t}$ is the sum of NW resistance, $R_{N W}$, total contact resistance, $R_{C}$, lead resistances, $R_{\text {lead }}$, and geometrical contributions, $R_{\text {geom }}$, due to current crowding effects,

$$
R_{\text {tot }}=\frac{4 \rho_{N W} L_{C}}{\pi d^{2}}+R_{C}+R_{\text {lead }}+R_{\text {geom }},
$$

where $R_{C}$ defines either the source or drain electrode/NW interface resistance depending on the bias. ${ }^{19}$

Typical NW resistances were of the order of several $\mathrm{M} \Omega$ up the G $\Omega$ range. $R_{\text {lead }}$ measured in short-circuited structures were less than $1 \mathrm{k} \Omega$, and $R_{\text {geom }}$ evaluated from a lateral current crowding model for cylindrical NWs introduced in an earlier work of ours ${ }^{23}$ was found to be two orders of magnitude smaller than $R_{C}$ and $R_{N W} \cdot{ }^{19}$

The specific contact resistivity, $\rho_{C}=R_{C} A_{C}$, of the metal-electrode/NW interface was calculated within the Transmission Line Model $^{22}$ using the interface area $A_{C}=\pi \beta d \Delta$ for $\beta \sim 0.5-0.75$ corresponding to the fraction of NW circumference covered with electrode metal. In all NW samples studied, $\rho_{C}$ increases with $\rho_{N W}$ (Fig. 3(a)) but decreases towards higher $N_{d}$ values (Fig. 3(b)), which is a signature of the lowering of the potential-barrier at the contact with augmenting $N_{d}$. A separation of $\rho_{C}$ values into different branches is observed following the different workfunctions of the electrode metals for both NW types. This result unambiguously demonstrates the suppression of Fermi level pinning in side-contacted Ge NW devices. The fact that the same effect is observed for two types of differently grown NWs proves that the effect is of general nature.

More intriguingly, for $\mathrm{Ag}$ contacts $\rho_{C}$ shows a linear $\log$-log dependence on $N_{d}$ indicating ohmic character of the interface ${ }^{24}$ (Fig. 3(b)). On the other hand, Pd and Au contacts give Schottky-type non-linear $\log -\log \rho_{C}-N_{d}$ dependence. ${ }^{24}$ The observed scaling of $\rho_{C}$ with $\rho_{N W}$ and $N_{d}$ (Fig. 3) suggests that the Pt contacts are of Schottky type as well. Numerical analysis of the $I-V$ characteristics of these devices confirms this conclusion. ${ }^{19}$

To gain insight into the extent of energy level realignment in Ge NW devices with the Schottky-type Au, Pd, and
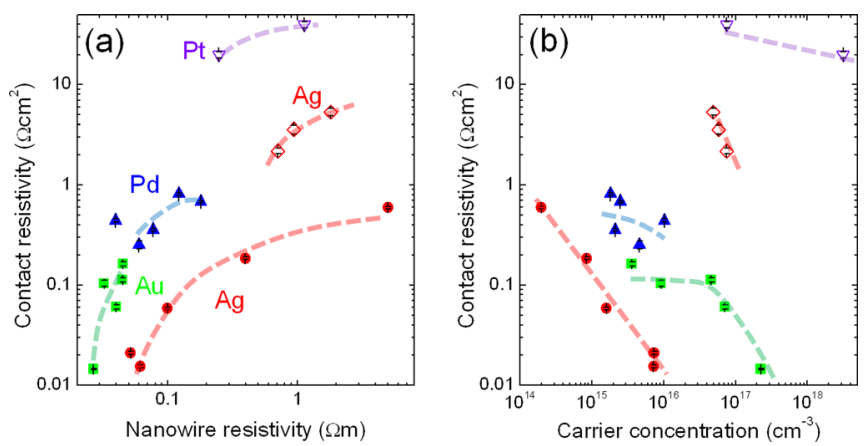

FIG. 3. Contact resistivity values plotted as function of (a) NW resistivity and (b) carrier concentration. Full and open symbols denote type A and type B NWs, respectively. Linear log-log dependence of contact resistivity on carrier concentration in the case of $\mathrm{Ag}$ contacts indicates ohmic character of the contact-interface. 

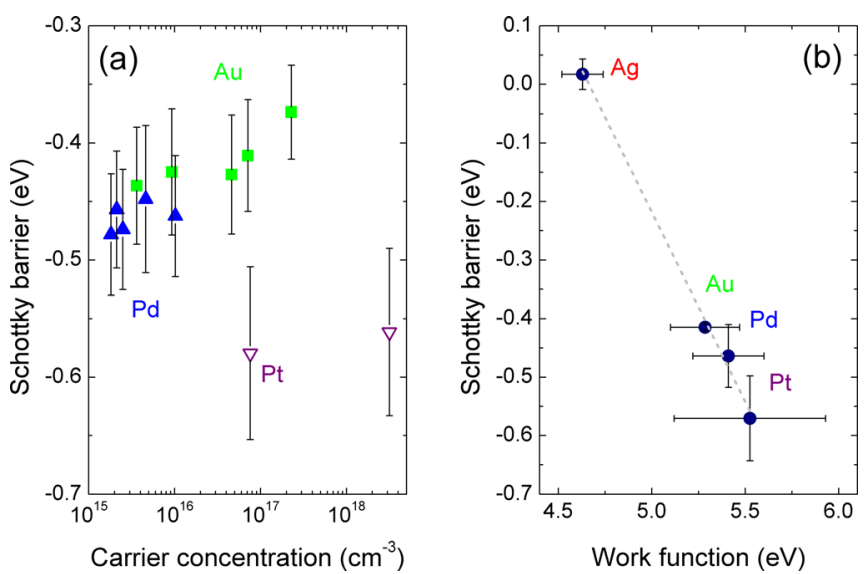

FIG. 4. (a) Schottky barrier heights for $\mathrm{Au}, \mathrm{Pd}$, and Pt contacts showing weak decrease with carrier concentration associated with image-field-force assisted barrier lowering. (b) Average measured Schottky barrier heights vs. metal work function. Linear fitting yields the pinning parameter $\gamma=0.65 \pm 0.03$, which indicates an exceptionally strong depinning of the Fermi level in side-contacted Ge NW devices.

Pt contacts, the potential barrier height, $\Phi_{S B}$, was estimated using the Thermionic Emission (TE) model, ${ }^{24}$

$$
\Phi_{S B}=k_{B} T \ln \frac{q \rho_{C} A^{*} T^{2}}{k_{B} T},
$$

with the elementary charge $q$, Boltzmann constant $k_{B}$, and the Richardson constant $A^{*}$ in $p$-type Ge equalling $3.48 \times 10^{5} \mathrm{~A} \mathrm{~m}^{-2} \mathrm{~K}^{-2}$ at temperature $T=300 \mathrm{~K}$. Based on our data, we excluded tunnelling and electron-hole recombination processes. ${ }^{19}$ For the ohmic case (Ag contacts), $\Phi_{S B}$ was calculated evaluating the relationship ${ }^{25}$

$$
\rho_{C} \propto \exp \left(-\frac{\Phi_{S B}}{\hbar} \sqrt{\frac{\varepsilon_{s} \varepsilon_{0} m^{*}}{N_{d}}}\right),
$$

with the effective hole mass $m^{*}=0.28 m_{0}, m_{0}$ being the free electron mass, $\varepsilon_{s}=16, \varepsilon_{0}$ the dielectric, and $\hbar$ the reduced Planck constant. $\Phi_{S B}$ for $\mathrm{Ag}$ contacts was found to be $0.02 \pm 0.01 \mathrm{eV}$, which lies in the range predicted for unpinned $\mathrm{Ag} / \mathrm{Ge}$ interfaces. ${ }^{19,26}$

The estimated $\Phi_{S B}$ values for $\mathrm{Au}, \mathrm{Pd}$, and Pt contacts (Fig. 4(a)) are negative, which is characteristic for depletion (Schottky) contacts to $p$-type semiconductors. ${ }^{24}$ A weak decrease of $\Phi_{S B}$ towards higher $N_{d}$ is due to a built-in potential resulting from the curvature of the contact-metal/NW interface. ${ }^{14}$ The pinning factor $\gamma \equiv\left|\frac{\partial \Phi_{S B}}{\partial \Phi_{m}}\right|=0.65 \pm 0.03$ (Fig. 4(b)) is significantly larger than 0 (fully pinned systems), in unambiguous agreement with Fermi level pinning suppression already shown by the data in Fig. 3 .

Our study provides experimental evidence of Fermilevel-pinning alleviation in side-contacted semiconductor NWs - a direct consequence of a transition from planar to 1D-confined channel geometry. The results demonstrate that the use of NW materials offers ways for band-engineering at metal/semiconductor interfaces not possible in bulk. Furthermore, the active conduction-channel and gate-length in the NW devices was found to extend below the full contact-electrode. The findings are in agreement with our earlier study on metallic $\mathrm{NWs},{ }^{23}$ demonstrating a general trend in quasi-1D nanomaterials.

The authors thank Dermot Daly (CRANN Advanced Microscopy Laboratory) for FIB lamella preparation help, TEM imaging and EDX spectroscopy. This work was supported by the Science Foundation Ireland contract-number PI-award 08/IN.1/I1873 and contract-number CSET 08/CE/ I1432. Part of this work was supported by the Cycle 4 of the Programme for Research in Third-Level Institutions (PRTLI4), Ireland.

${ }^{1}$ C. M. Lieber, MRS Bull. 36, 1052 (2011).

${ }^{2}$ P. Yang, R. Yan, and M. Fardy, Nano Lett. 10, 1529 (2010).

${ }^{3}$ S. Ju, A. Facchetti, X. Xuan, J. Liu, F. Ishikawa, P. D. Ye, C. Zhou, T. J. Marks, and D. B. Janes, Nature Nanotechnol. 2, 378 (2007).

${ }^{4}$ J. Xiang, W. Lu, Y. Hu, H. Yan, and C. M. Lieber, Nature 441, 489 (2006).

${ }^{5}$ X. Wu, J. S. Kulkarni, G. Collins, N. Petkov, D. Almecija, J. J. Boland, D. Erts, and J. D. Holmes, Chem. Mater. 20, 5954 (2008).

${ }^{6}$ F. Leonard and A. A. Talin, Nat. Nanotechnol. 6, 773 (2011).

${ }^{7}$ V. Chaudhry, E. Ramamurthi, E. Fong, and M. S. Islam, Nano Lett. 7, 1536 (2007).

${ }^{8}$ E. Stern, G. Cheng, M. P. Young, and M. A. Reed, Appl. Phys. Lett. 88, 053106 (2006).

${ }^{9}$ J. Salfi, U. Philipose, C. F. de Sousa, S. Aouba, H. E. Ruda, Appl. Phys. Lett. 89, 261112 (2006).

${ }^{10}$ S. E. Mohney, Y. Wang, M. A. Cabassi, K. K. Lew, S. Dey, J. M. Redwing, and T. S. Mayer, Solid State Electron. 49, 227 (2005).

${ }^{11}$ International Technology Roadmap for Semiconductors: Emerging Research Materials (ITRS, 2011).

${ }^{12}$ A. Cathignol, B. Cheng, D. Chanemougame, A. R. Brown, K. Rocherau, G. Ghibaudo, and A. Asenov, IEEE Electron. Devices Lett. 29, 609 (2008).

${ }^{13}$ F. Leonard and A. A. Talin, Phys. Rev. Lett. 97, 026804 (2006).

${ }^{14}$ C. Malagu, V. Guidi, M. C. Carotta, and G. Martinelli, Appl. Phys. Lett. 91, 808 (2002).

${ }^{15}$ H. Statz, G. A. deMars, L. Davis, and A. Adams, Jr., Phys. Rev. 101, 1272 (1956).

${ }^{16}$ T. Nishimura, K. Kita, and A. Toriumi, Appl. Phys. Lett. 91, 123123 (2007).

${ }^{17}$ L. T. Ngo, D. Almecija, J. E. Sader, B. Daly, N. Petkov, J. D. Holmes, D. Erts, and J. J. Boland, Nano Lett. 6, 2964 (2006).

${ }^{18}$ G. Collins, M. M. Koleśnik, V. Krstić, and J. D. Holmes, Chem. Mater. 22, 5235 (2010).

${ }^{19}$ See supplementary material at http://dx.doi.org/10.1063/1.4821996 for further details on experimental procedures, analysis, and models used.

${ }^{20}$ D. Josell, S. H. Brongersma, and Z. Tokei, Annu. Rev. Mater. Res. 39, 231 (2009).

${ }^{21}$ CRC Handbook of Chemistry and Physics, 85th ed. (CRC Press, Boca Raton, FL, 2004-2005).

${ }^{22}$ H. H. Berger, Solid State Electron. 15, 145 (1972).

${ }^{23}$ M. M. Koleśnik, S. Hansel, T. Lutz, N. Kinahan, M. Boese, and V. Krstić, Small 7, 2873 (2011).

${ }^{24}$ A. Y. C. Yu, Solid State Electron. 13, 239 (1970).

${ }^{25}$ Z. Zhang, K. Yao, Y. Liu, C. Jin, X. Liang, Q. Chen, Adv. Funct. Mater. 17, 2478 (2007).

${ }^{26}$ S. M. Sze and K. K. Ng, Physics of Semiconductor Devices, 3rd ed. (Wiley, New York, 2007). 